\title{
Idades U-Pb (LA-ICPMS) para as suítes Caraguataí e Jussiape, Bloco Gavião, Bahia
}

\author{
Simone Cerqueira Pereira Cruz ${ }^{1}$, Maurício Antônio Carneiro ${ }^{2}$, Johildo Salomão Figueiredo \\ Barbosa $^{3}$, Adriano Alberto Marques Martins ${ }^{4}$, Jocilene dos Santos Santana ${ }^{3}$ Leo Teixeira ${ }^{4}$, \\ Guilherme de Souza Silva Tavares Gonçalves, Angela Beatriz Menezes Leal ${ }^{3}$, Elton Dantas ${ }^{5}$ \\ \& Márcio Pimentel ${ }^{5}$
}

\begin{abstract}
Resumo A área de trabalho encontra-se inserida na porção nordeste do Cráton do São Francisco, no Bloco Gavião. O mapeamento geológico realizado na região de Abaíra-Jussiape permitiu identificar duas suítes magmáticas, que foram denominadas de Caraguataí e Jussiape. Ambas as suítes encontram-se truncadas por zonas de cisalhamento reversas a reversa-destrais que levaram à formação de rochas da série milonítica. A suíte Caraguataí, mais antiga, compreende álcali-feldspato granitos, sienitos e quartzo-sienitos, cujo componente mesomilonítico foi datado pelo método U-Pb (LA-ICPMS) em $2711 \pm 13$. A Suíte Jussiape, intrusiva na primeira, é constituída por monzogranitos a sienogranitos, que foram datados em $2076 \pm 51 \mathrm{Ma}$. Esses dados sugerem a existência de magmatismo neoarqueano e paleoproterozóico nesse setor do Bloco Gavião.
\end{abstract}

Palavras-chave: Zonas de cisalhamento, anticlinal, Corredor do Paramirim, Bloco Gavião.

\begin{abstract}
U-Pb ages(LA-ICPMS) for the Caraguatai and Jussiape Suites, São Francisco Craton, Gavião Block, Bahia, Brazil. The study area is inserted in the northeastern portion of the São Francisco Craton, in the Gavião Block. The geological mapping carried out in the Abaíra-Jussiape region allowed us identify two magmatic suites named Caraguataí and Jussiape. Both suites are crosscut by reverse to reversedextral shear zones, which resulted in the formation of mylonitic rocks. The older Caraguataí Suite includes alkali-feldspar granites, syenites and quartz-syenites, whose mesomylonitic component was dated by U-Pb method (LA-ICPMS) at $2711 \pm 13$ Ma. The Jussiape Suite, intrusive in the former, consists of monzogranites to syenogranites, which were dated at $2076 \pm 51 \mathrm{Ma}$. These ages suggest the existence of Neoarchean and Paleoproterozoic magmatism in this sector of the Gavião Block.
\end{abstract}

Keywords: Shear zones, anticline, Paramirim Corridor, Gavião Block.

INTRODUÇÃO Na região de Abaíra-Jussiape (Fig. 1), no domínio morfológico da Chapada Diamantina, aflora uma janela estrutural de rochas do embasamento de idade arqueano-paleoproterozóica, cuja exposição está relacionada com a presença de uma culminação antiformal envolvendo a cobertura do Supergrupo Espinhaço (Guimarães et al. 2005). Essa região vem sendo mapeada geologicamente (Lopes 1991, Guimarães et al. 2005) e estudada litogeoquímica(Teixeira 2005), geocronológica- (Guimarães et al. 2005) e estruturalmente (Cruz 2004).

Este trabalho apresenta resultados geocronológicos e isotópicos obtidos a partir dos dois principais componentes do substrato siálico da região de Abaíra-Jussiape representados pelas suítes intrusivas Caraguataí (originalmente denominada de Complexo Caraguataí por Lopes 1991) e Jussiape. A ausência de dados geocronológicos de precisão nesta região motivou o estudo sistemático de datação pelos métodos geocronológicos U-Pb em zircão por Laser AblationICPMS e Sm-Nd das rochas da região em foco. Com isso, pretende-se contribuir para o entendimento da evolução tectônica do embasamento da porção nordeste do Cráton do São Francisco, que representa um dos segmentos crustais mais antigos da plataforma sulamericana, com idades isotópicas variando entre 3,5 e 2,1 Ga (Leal 1998, Cunha et al. 1996). Para obtenção desses resultados foram utilizadas as técnicas de datação $\mathrm{U}-\mathrm{Pb}$ por LA-ICPMS (Laser Ablation - Inductively Coupled Plasma Mass Spectrometry) e idades modelo Sm-Nd $\mathrm{T}_{\mathrm{DM}}$ por TIMS (Thermal Ionization Mass Spectrometry).

CONTEXTO GEOLÓGICO REGIONAL OBloco Gavião (sensu Barbosa \& Sabaté 2002) (Fig. 2) ocorre na porção setentrional do Cráton do São Francisco e

1 - NGB-PPGG-IGEO-UFBA, Salvador (BA), Brasil. E-mail: simonecruzufba@gmail.com

2 - DEGEO/EM/UFOP, Ouro Preto (MG), Brasil. E-mail: mauricio@degeo.ufop.br

3 - UFBA, Salvador (BA), Brasil. E-mails: johildo@cpgg.ufba.br; jocilenesanttana@yahoo.com.br; guigaufba@yahoo.com.br; angelab@ufba.br

4 - CPRM. E-mails: adriano1952@gmail.com ; leort@sa.cprm.gov.br

5 - UnB, Brasília (DF), Brasil. E-mails: elton@unb.br; marcio@unb.br 


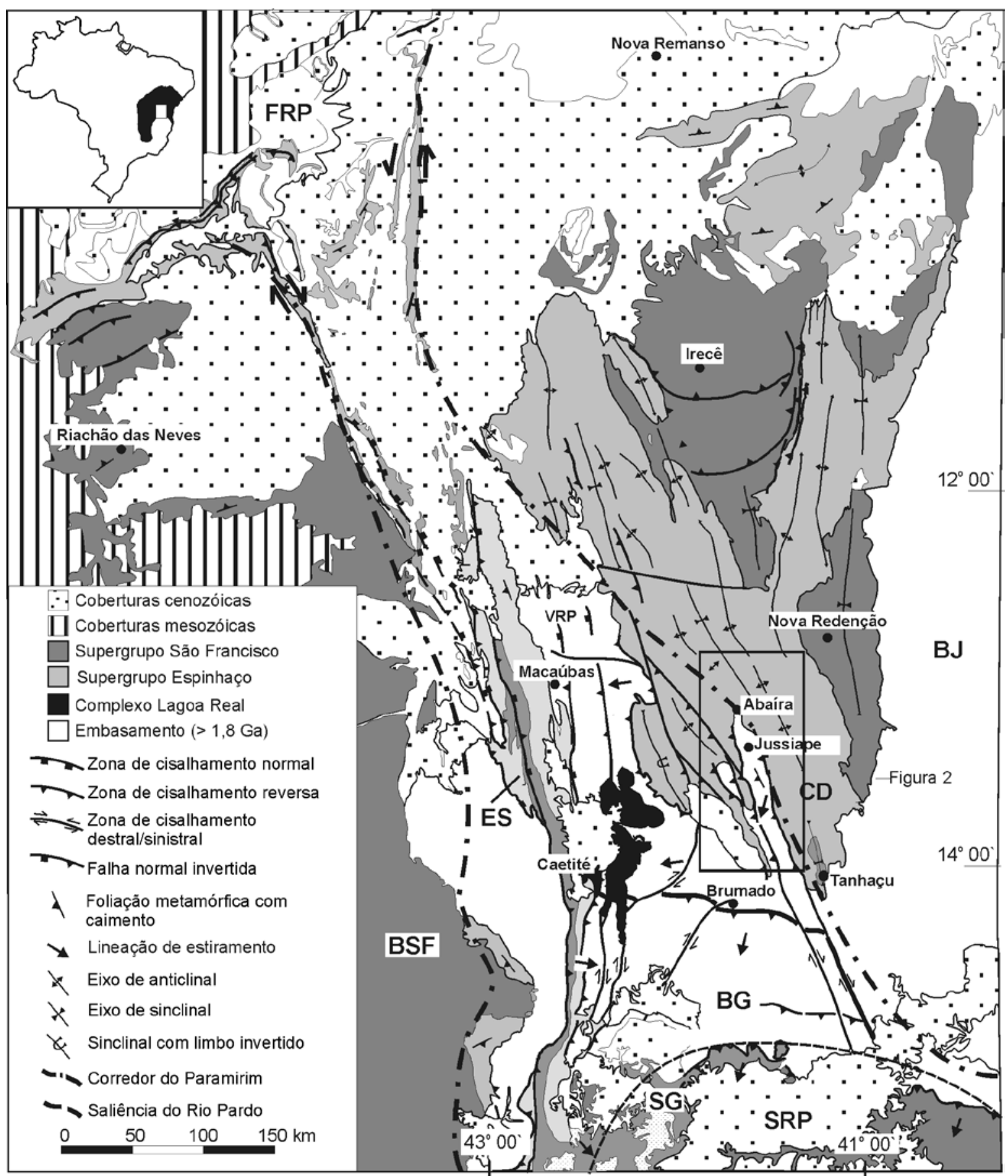

Figura 1 - Mapa geológico regional simplificado que compreende o Bloco Gavião, granitóides paleoproterozóicos do Complexo Lagoa Real, assim como as coberturas proterozóicas dos supergrupos Espinhaço e São Francisco. O retângulo representa a área da Anticlinal de Abairra-Jussiape. BG - Bloco Gavião, BJ - Bloco Jequié, ES - Espinhaço Setentrional, CD - Chapada Diamantina, FRP - Faixa Rio Preto, SRP - Saliencia do Rio Pardo (Faixa Araçuaí), VRP - Vale do Rio Paramirim, BSF - Bacia do São Francisco e SG - Serra Geral. Modificado de Cruz (2004).

é constituído por gnaisses de composição tonalíticagranodiorítica-granítica, migmatitos, anfibolitos, granulitos, de idade arqueana-paleoproterozóica (Cunha \& Fróes 1994, Leal et al. 1998) e remanescentes de seqüências meta-vulcanossedimentares arqueanas agrupadas em Contendas Mirantes, Mundo Novo, Ibitira-Ubiraçaba, Brumado, Guajeru, Riacho de
Santana, Urandi, Boquira e Licínio de Almeida (Silva \& Cunha 1999) (Cunha et al. 1996, Leal et al. 1998, Silva \& Cunha 1999, Arcanjo et al. 2000).

$\mathrm{O}$ conjunto de dados $\mathrm{U} / \mathrm{Pb}, \mathrm{Rb} / \mathrm{Sr}$ e $\mathrm{Pb} / \mathrm{Pb}$ obtidos em rochas do Bloco Gavião sugere que a formação desse compartimento está relacionada com três eventos de plutonismo arqueanas ocorridos em 


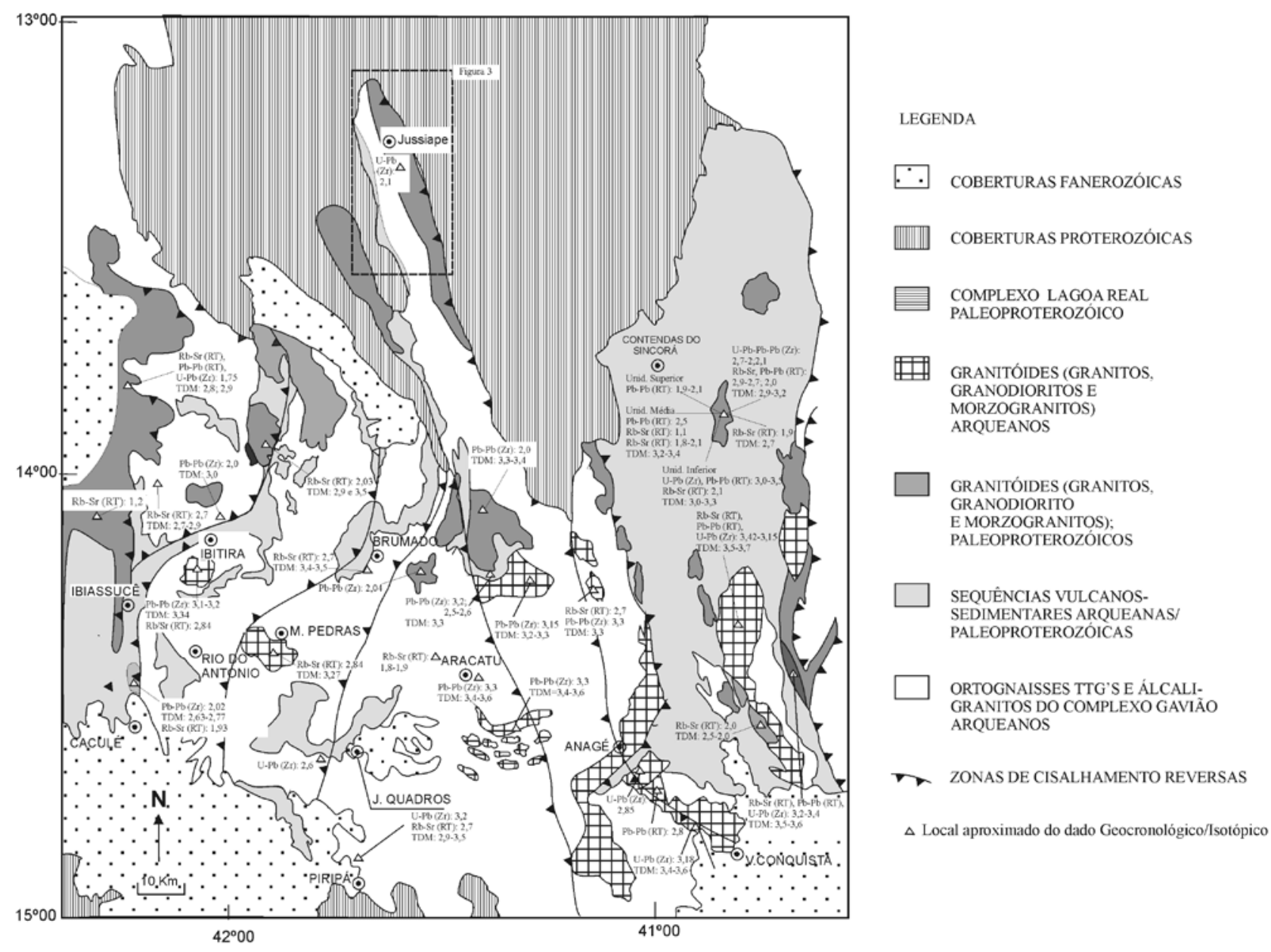

Figura 2 - Esboço geológico da porção Sul do Bloco Gavião (Modificado de Leal 1998). As idades estão em bilhões de anos (Ga). RT-Rocha Total, Zr - Zircão. Mapa baseado em: 1-Cordani \& Iyer (1979); 2Marinho et al. (1979); 3- Cordani et al. (1985); 4- Turpin et al. (1988); 5-Wilson et al. (1988); 6- Mascarenhas \& Garcia (1989); 7- Sabaté et al. (1990); 8- Marinho (1991); 9- Martin et al. (1991); 10-Cordani et al. (1992); 11-Ledru et al. (1993); 12-Nutman \& Cordani (1993); 13- Nutman et al. (1993); 14- Pimentel et al.(1994); 15- Rosa et al. (1996); 16- Barbosa \& Dominguez (1996); 17-Leal et al. (1997); 18-Cordani et al. (1997); 19- Martin et al. (1997); 20- Pinto (1996); 21-Leal et al. (1998); 22- Sato (1998), 23-Leal (1998); 24-Leal et al. (2000); 25-Lopes (2002); 26-Leal et al. (2003); 27- Guimarães et al. (2005) ; 28- Marinho et al. (2008). O retângulo indica a localização da figura 3.

3.4-3.2 Ga, 3.2-3.1 Ga and 2.9-2.7 Ga (Cordani et al. 1985, 1992; Martin et al. 1991, Marinho 1991, Nutman \& Cordani 1993, Cunha et al. 1996, Pinto 1996, Leal 1998, Santos-Pinto et al. 1998 em preparação; Peucat et al. 2002). Um evento de plutonismo granítico de idade riaciana-orosiriana também vem sendo apresentado por diversos autores (Santos-Pinto et al. 1998, Arcanjo et al. 2000, Leal et al. 2000, Leal et al. 2005, Rosa et al. 1996, Guimarães et al. 2005).

No Bloco Gavião afloram coberturas proterozóicas dos supergrupos Espinhaço e São Francisco. O Supergrupo Espinhaço, de idade estateriana-esteniana, compreende vulcanitos ácidos na base, que são sucedidos por rochas siliciclásticas. $\mathrm{O}$ inicio da sua deposição marca a fase da geração de riftes intracontinentais, cuja abertura inicial levou à colocação de vulcânicas ácidas que foram datadas de $1.752 \pm 4 \mathrm{Ma}, 1.748 \pm 4 \mathrm{Ma}$ e $1.731 \pm 5 \mathrm{Ma}(\mathrm{U}-\mathrm{Pb}$ em zircão) obtidas, respectivamente, por Schobbenhaus et al. (1994), Babinski et al. (1999) e Danderfer et al. (2009). Essas rochas estão cortadas por diques máficos gabróicos e toleíticos, distinguidos em dois grupos: Grupo I: $1492 \pm 16 \mathrm{Ma}$ (Loureiro et al. 2010); $1514 \mathrm{Ma}$ (Babinsnky et al. 1999) e $1496 \mathrm{Ma}$ (Guimarães et al. 2005); Grupo II- $854 \pm 23$ Ma (Danderfer et al. 2009) e $834 \mathrm{Ma}$ (Loureiro et al. 2010). O supergrupo São Francisco, de idade neoproterozóica, engloba na base diamictitos glaciogênicos que gradam para carbonatos e pelitos de ambiente marinho (Guimarães 1996).

A GEOLOGIA NA REGIÃo DE ABAÍRAJUSSIAPE Como unidades constituintes do Bloco Gavião na região em foco, têm-se três grupos principais de rochas (Fig. 3): (i) o Complexo Brumado; (ii) os álcali-feldspato granitos, sienitos e quartzo-sienitos miloníticos da Suíte Caraguataí; e (iii) o granito da 


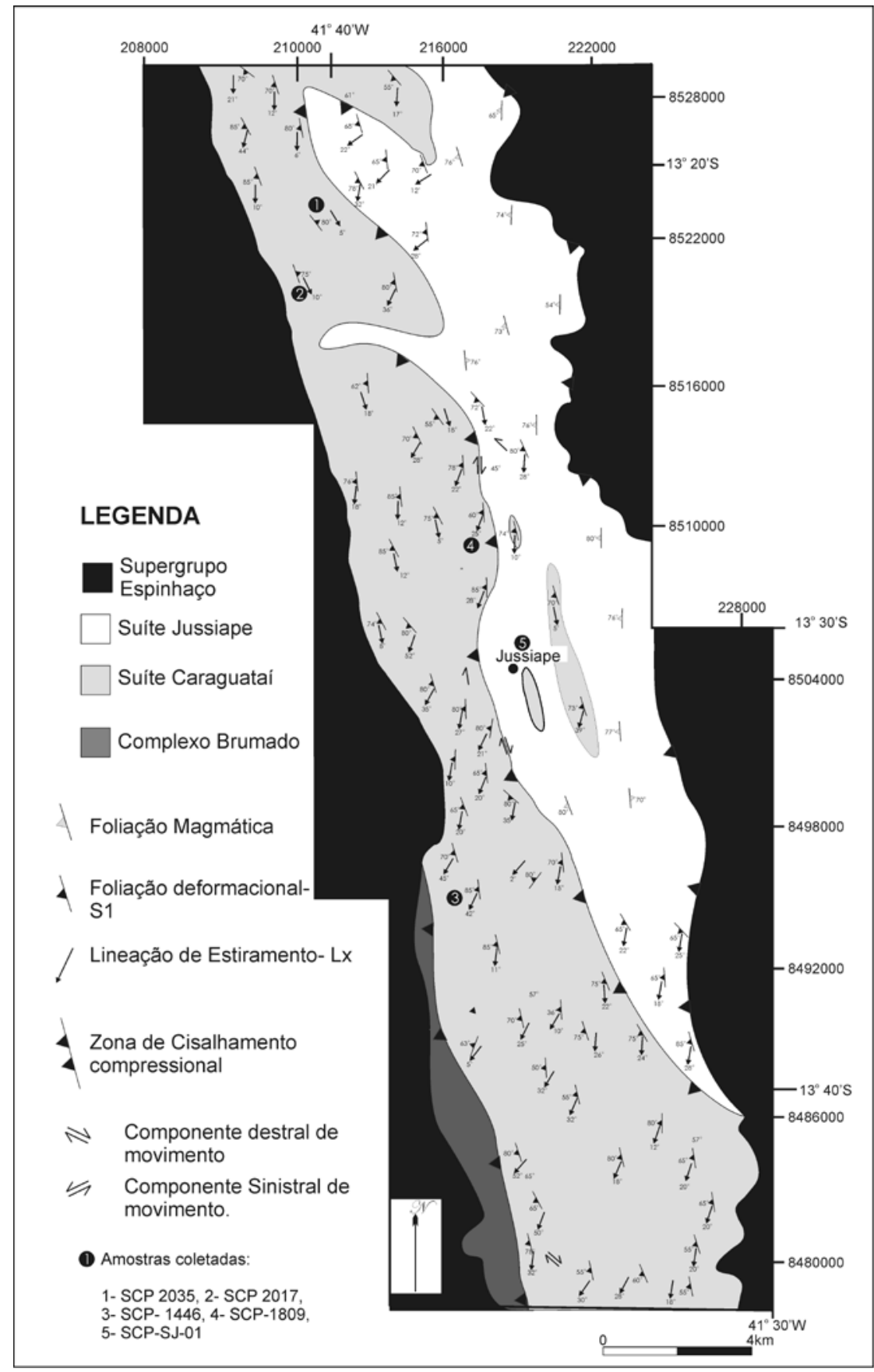

Figura 3 - Mapa simplificado do núcleo da Anticlinal de Abaíra Jussiape com ênfase nas principais suites aflorantes, com a localização das amostras coletadas para o estudo isotópico. A localização da figura está indicada na figura 2.

Suíte Jussiape, milonitizado ou não. O Complexo Brumado é constituído por metabasaltos (Fig. 4a) com metaultramafitos associados, metandesito, metarritmito e metarenitos (Guimarães et al. 2005) todos milonitizados e não representa objeto de estudo nesse trabalho. Guimarães et al. (2005) consideraram o complexo em questão como sendo de idade meso a neoarqueana.

$\mathrm{Na}$ Suíte Caraguataí ocorrem álcali-feldspato granitos, sienitos/quartzo-sienitos miloníticos são rochas leuco a mesocráticas, de coloração cinza. O protólito ígneo dessas rochas foi submetido a graus variáveis deformações emzonas de cisalhamento destrais a destrais reversas, dando origem a rochas protomiloníticas, augen-mesomiloníticas e ultramiloníticas (Fig. 4b). Os domínios de menor deformação gradam para os de maior deformação na escala de afloramento. Neste sentido, as rochas protomiloníticas apresentam porfiroclastos com diâmetros médios em torno de 4 $\mathrm{cm}$, podendo alcançar $8 \mathrm{~cm}$. Nessas rochas a foliação 
marcada pela biotita e, em alguns casos, anfibólio, ainda é incipientemente desenvolvida e descontínua. Do ponto de vista litogeoquímico, os protólitos da Suíte Caraguataí foram classificados por Teixeira (2005) como rochas metaluminosas a peraluminosas, ricas em ferro, alcalinas e de ambiente intraplaca continental. De acordo com esse autor, o empobrecimento de $\mathrm{Sr}$ acompanhado de enriquecimento de $\mathrm{Y}$ indica uma gênese a partir de uma fusão parcial em região onde o plagioclásio era estável, ou seja, a pressão inferior a 10 kbar (menos de $30 \mathrm{~km}$ de profundidade) e em condições de elevado gradiente geotérmico.

A Suíte Jussiape é formada por monzogranitos a sienogranitos, milonitizados ou não, hololeucocráticos a leucocráticos, de coloração rósea. Nessa Suíte foram reconhecidas duas litofácies: (i) fanerítica média (Fig. 5); e (ii) porfirítica média. Na fácies fanerítica média, as rochas são isotrópicas, equigranulares com textura granoblástica. $\mathrm{Na}$ fácies porfirítica, por sua vez, predominam fenocristais de feldspato alcalino, com granulação média em torno de $2 \mathrm{~cm}$, podendo alcançar até $3 \mathrm{~cm}$. As rochas dessa fácies comumente exibem estruturas de fluxo magmático, cuja orientação geral é $262^{\circ} / 76^{\circ}$.

Do ponto de vista litogeoquímico, Teixeira (2005) classificou as rochas da Suíte Jussiape como muito diferenciadas, peraluminosas, cálcio-alcalino de alto $\mathrm{K}$ e relacionados com um magmatismo do tipo I, em condições pós-orogênica, cujas características químicas são bem próximas às de um magmatismo tipo A.

\section{APRESENTAÇÃO E DISCUSSÃO DOS RESULTADOS U-PB (LA-ICPMS) A localização} das amostras selecionadas para os estudos isotópicos apresentados neste trabalho pode ser observada na figura 3 e os resultados analíticos nas tabelas 1 e 2 e figuras 6 e 7. O material coletado para as análises $\mathrm{U}-\mathrm{Pb}$ foi preparado no Laboratório de Preparação de Amostras (LOPAG) da Universidade Federal de Ouro Preto. Nesse laboratório, as amostras foram britadas, pulverizadas e levadas para extração de frações de minerais

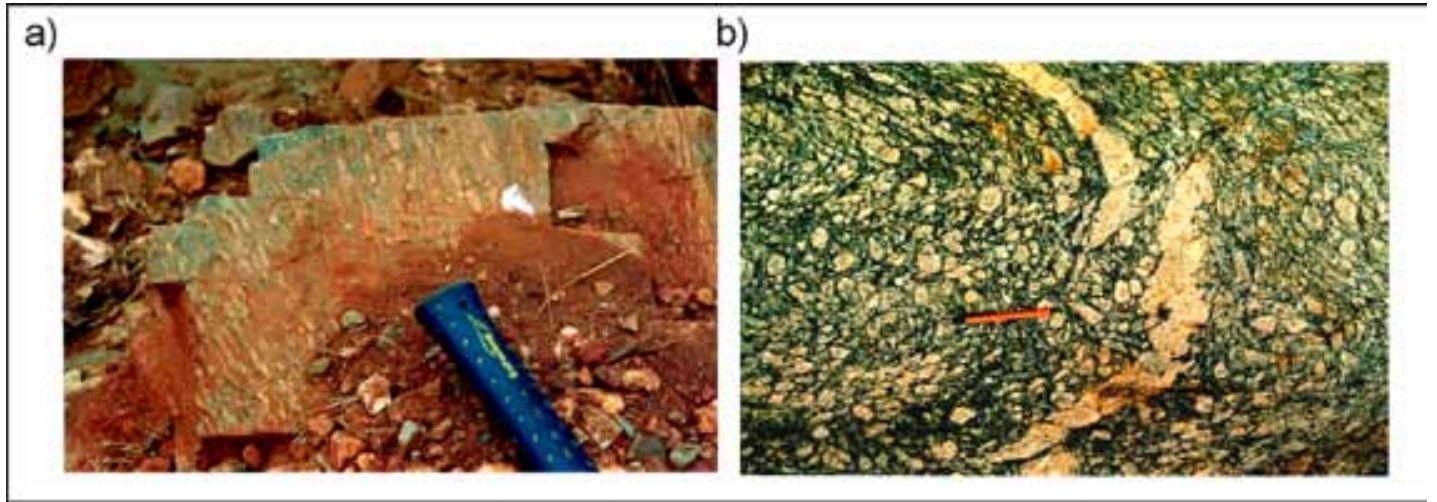

Figura 4 - Metabasaltos foliados do Complexo Brumado que afloram na porção oeste da área de trabalho(a); augen mesomilonito da Suite Caraguatai (b).

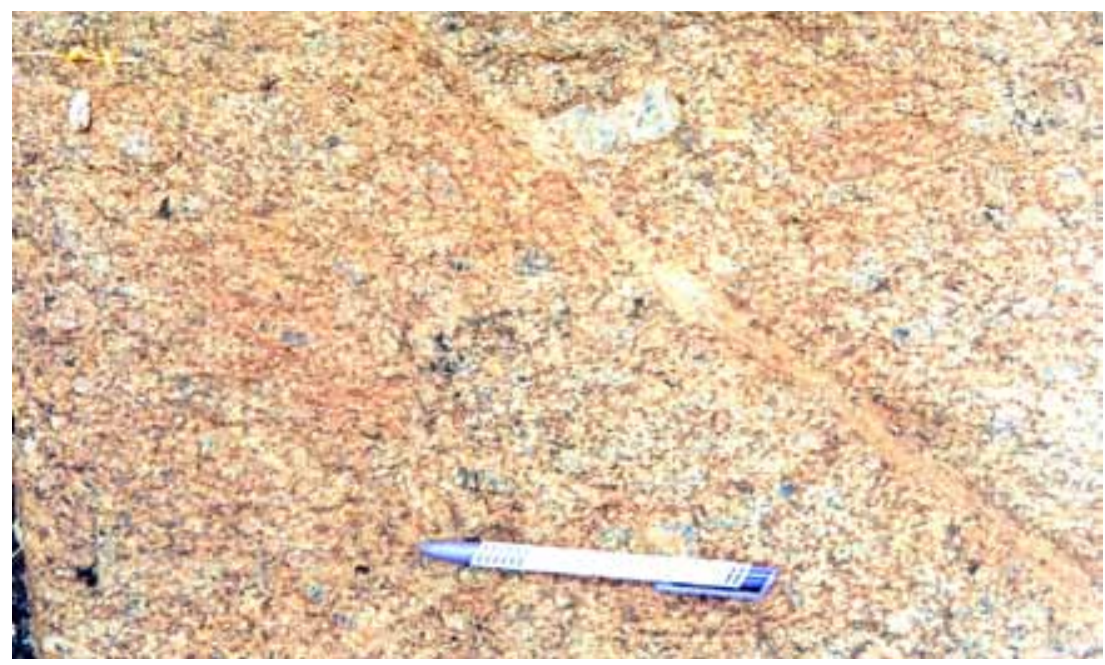

Figura 5 - Aspecto macroscópico da fácies fanerítica média, leucrocrática da Suíte Jussiape. 
Tabela 1 - Principais dados geocronológicos para as rochas do Bloco Gavião e unidades vizinhas. Modificado de Leal (1998). 1-Cordani \& Iyer (1979); 2- Marinho et al. (1979); 3- Cordani et al. (1985); 4- Turpin et al. (1988); 5- Wilson et al. (1988); 6- Mascarenhas \& Garcia (1989); 7- Sabaté et al. (1990); 8- Marinho (1991); 9- Martin et al. (1991); 10-Cordani et al. (1992); 11-Ledru et al. (1993); 12- Nutman \& Cordani (1993); 13- Nutman et al. (1993); 14- Pimentel et al.(1994); 15- Rosa et al. (1996); 16- Barbosa \& Dominguez (1996); 17- Leal et al. (1997); 18-Cordani et al. (1997); 19-Martin et al. (1997); 20- Pinto (1996); 21 - Leal et al. (1998); 22- Sato (1998), 23-Leal (1998); 24-Leal et al. (2000); 25-Lopes (2002); 26- Leal et al. (2003); 27- Guimarães et al. (2005); 28- Marinho et al. (2008).

\begin{tabular}{|c|c|c|c|c|c|c|}
\hline Unidades Geológicas & U-Pb e 207Pb/206Pb (Ma) & Rb-Sr (Ga) & $\begin{array}{c}207 \mathrm{~Pb} / 206 \mathrm{~Pb} \\
(\mathrm{Ga}) * * *\end{array}$ & $\begin{array}{l}\text { Sm-Nd } \\
\text { (TDM) } \\
(\mathrm{Ga})\end{array}$ & $\begin{array}{l}\text { K-Ar } \\
\text { (Ga) }\end{array}$ & Referências \\
\hline Granitóide Sete Voltas & $3403,3243+/-5,3158+/-2 *$ & $\begin{array}{l}3,4 ; 3,2 ; 3,1 \\
2,6^{* * *}\end{array}$ & $3,2 * * *$ & $3,7-3,5$ & $1,9-1,8$ & $\begin{array}{c}3,6,9,12 \\
19\end{array}$ \\
\hline Gnaisse Boa Vista/Mata Verde & $3.353+/-5 *$ & $3,3 * * *$ & $3,4 * * *$ & $3,5-3,2$ & $0,9-0,4$ & $3,5,6,8,12$ \\
\hline Tonalito de Bernarda & $3332+/-4 *$ & $2,6-2,7 * * *$ & & $3,3-3,5$ & & 3,20 \\
\hline $\begin{array}{c}\text { Granitóides de Mariana e } \\
\text { Aracatu }\end{array}$ & $\begin{array}{c}3245+/-25 *, 3250+/-4 *, 3240+/- \\
14 \mathrm{Ma}^{*}, 3.325+/-10^{*}, \\
2506+/-10 *, 1944+/-7 * \\
\end{array}$ & $0,5^{* * *}$ & & $3,6-3,5$ & $0,6-0,5$ & 20 \\
\hline Gnaisses de Piripá & $3200+/-5^{*}$ & 2,7 e $2,0 * * *$ & & $3,5-2,9$ & 0,6 & $6,18,22$ \\
\hline Granitóides Lagoa do Morro & $3184+/-6^{*}$ & 3,2 e $2,8 * * *$ & $2,8 * * *$ & $3,6-3,3$ & $0,6-0,5$ & $2,3,7,8,9$ \\
\hline Granitos Serra do Eixo & $\begin{array}{c}3158+/-5 *, 2,5-2,6^{*}, \\
2695+/-10^{*}\end{array}$ & & & 3,3 & & 20 \\
\hline Granito Serra dos Pombos & $2845+/-45 *$ & $2,8^{* * *}$ & $2,8 * * *$ & & & 8,12 \\
\hline $\begin{array}{l}\text { Rochas Gnáisicas e } \\
\text { Migmatíticas }\end{array}$ & $3200+/-11^{*}$ & $\begin{array}{c}2,7 \text { e } 1,9-1,8 \\
* * *\end{array}$ & & $3,4-3,1$ & $0,6-0,5$ & $6,17,22$ \\
\hline Granito Malhada de Pedra & & $2,8 * * *$ & & 3,27 & & 17,21 \\
\hline Granito Lagoa da Macambira & $3146+/-24 *$ & $2,840 * * *$ & $3,1-3,2 * * *$ & 3,34 & & 21,23 \\
\hline Granitos alcalinos Serra do Eixo & $2500-2600 *$ & & & 3,3 & & 17,20 \\
\hline Sill do Rio Jacaré & & $1,9^{* * *}$ & $2,5 * * *$ & $3,5-3,3$ & & 6,8 \\
\hline $\begin{array}{l}\text { Granitos sub-alcalinos e } \\
\text { alcalinos de Pé de Serra }\end{array}$ & $2652+/-11 *, 2282+/-81 *$ & $2,6-2,2,1,2 * * *$ & 2,$6 ; 2,3 * * *$ & $3,2-3,1$ & $2,0-1,8$ & $3,8,28$ \\
\hline Granito Umburanas & $2049+/-5^{*}$ & & & 3,3 & & 20 \\
\hline Granito Serra da Franga & $2039+/-11 *$ & & & & & 20 \\
\hline Granito Mariana & $3259+/-5 \mathrm{Ma} *, 1944+/-7 *$ & & & $3,5-3,6$ & & 20 \\
\hline Granito Aracatu & $\begin{array}{c}3240+/-1 *, 3.371+/-14^{*} \\
3.325+/-10^{*}, 2506+/-10^{*}, \\
2149+/-15^{*}, 1,95-2,26^{*}\end{array}$ & & & 3,6 & & $7,8,20$ \\
\hline Granito Gameleira & & $1,95 * * *$ & & $2,6-2,8$ & & 7,8 \\
\hline Granito Riacho Pedras & & $1,9 * * *$ & & 3,2 & & 7,8 \\
\hline $\begin{array}{c}\text { Granito Lagoa Grande/ } \\
\text { Lagoinha }\end{array}$ & & $2,0 * * *$ & & $2,7-2,9$ & & 7,8 \\
\hline Ganito Iguatemi & & $2,03 * * *$ & & $2,9-3,5$ & 0,48 & 24 \\
\hline Granito Rio do Paulo & & $1,96 * * *$ & & 2,73 & 0,50 & 24 \\
\hline Granito Espírito Santo & $2023+/-26 *, 997+/-32 *$ & $1,6 * * *$ & $2,01^{*}$ & $3,05-3,09$ & 0,49 & 24 \\
\hline Granito Caculé & $\begin{array}{c}2070+/-72 *, \\
1956+/-562070+/-72 *\end{array}$ & $1,9 * * *$ & $2,02 *$ & $2,63-2,77$ & $\begin{array}{c}1,06- \\
0,55\end{array}$ & 20,24 \\
\hline TTG Guajeru & 3191-3394* & & & & & 25 \\
\hline Granito Jussiape & $2122 *$ & & & & & 27 \\
\hline Granitóide de Veredinha & $2103 *$ & & & & & 27 \\
\hline Granitóide de Ibitiara & 2091* & & & & & 27 \\
\hline Granito Alcalino Guajeru & 2649-2660* & & & & & 25 \\
\hline $\begin{array}{c}\text { Contendas Mirante - Unidade } \\
\text { Superior }\end{array}$ & $2150-1900 *$ & & & & & 3,8 \\
\hline $\begin{array}{c}\text { Contendas Mirante -Unidade } \\
\text { Média }\end{array}$ & & 2,2 e $1,2 * * *$ & $2,5 * * *$ & $3,4-3,2$ & & 3,8 \\
\hline $\begin{array}{c}\text { Contendas Mirante -Unidade } \\
\text { Inferior } \\
\end{array}$ & & 2,5 e $2,0 * * *$ & & $3,3-3,0$ & $2,0-1,5$ & 3,8 \\
\hline Umburanas - Unidade Média & & $2,2 * * *$ & 2,7 e $2,0 * * *$ & & $1,0-05$ & 3,26 \\
\hline $\begin{array}{c}\text { Complexo Lagoa - Real - } \\
\text { Rochas Gnáissicas }\end{array}$ & & $2,7 * * *$ & & & & $4,10,14$ \\
\hline $\begin{array}{l}\text { Complexo Lagoa - Real - } \\
\text { Granito São Timotéo }\end{array}$ & $1746+/-5^{*}$ & $1,7 * * *$ & $1,7 * * *$ & $2,7-2,9$ & 0,5 & $4,10,14,22$ \\
\hline $\begin{array}{l}\text { Complexo Lagoa - Real - } \\
\text { Albititos }\end{array}$ & $1745+/-15 ; 961+/-22 *$ & $1,5^{* * *}$ & & & & $4,10,14$ \\
\hline
\end{tabular}

*Zircão, ** Monazita, *** Rocha Total (RT) 
Tabela 2 - Resultados U-Pb (LA-ICPMS) para as suites Caraguataí e Jussiape.

\begin{tabular}{|c|c|c|c|c|c|c|c|c|c|c|c|c|c|}
\hline Amostra & $\mathrm{Th} / \mathrm{U}$ & ${ }^{206} \mathrm{~Pb} /{ }^{238} \mathrm{U}$ & $\begin{array}{l} \pm 2 \sigma \\
(\%)\end{array}$ & ${ }^{207} \mathrm{~Pb} /{ }^{235} \mathrm{U}$ & $\begin{array}{l} \pm 2 \sigma \\
(\%)\end{array}$ & ${ }^{207} \mathrm{~Pb} /{ }^{206} \mathrm{~Pb}$ & $\begin{array}{l} \pm 2 \sigma \\
(\%)\end{array}$ & $\begin{array}{l}{ }^{206} \mathrm{~Pb}^{/ 238} \mathrm{U} \\
(\mathrm{Ma})\end{array}$ & \begin{tabular}{|l} 
$\pm 2 \sigma$ \\
$(\mathrm{Ma})$ \\
\end{tabular} & $\begin{array}{l}{ }^{207} \mathrm{~Pb} /{ }^{206} \mathrm{~Pb} \\
(\mathrm{Ma})\end{array}$ & $\begin{array}{l} \pm 2 \sigma \\
(\mathrm{Ma})\end{array}$ & $\begin{array}{l}{ }^{207} \mathrm{~Pb} /{ }^{205} \mathrm{~Pb} \\
(\mathrm{Ma})\end{array}$ & $\begin{array}{l} \pm 2 \sigma \\
\text { (Ma) }\end{array}$ \\
\hline \multicolumn{14}{|c|}{ Suíte Caraguataí } \\
\hline \multicolumn{14}{|c|}{ Amostra SCP-2017 } \\
\hline $03 \mathrm{Z1}$ & 0.45 & 0.48 & 9.28 & 12.23 & 9.97 & 0.19 & 3.64 & 2519 & 193 & 2704 & 60 & 2623 & 94 \\
\hline $04 \mathrm{Z2}$ & 0.40 & 0.56 & 7.66 & 14.33 & 8.52 & 0.19 & 3.72 & 2852 & 177 & 2714 & 61 & 2772 & 81 \\
\hline $05 \mathrm{Z3}$ & 0.46 & 0.48 & 8.54 & 12.40 & 9.43 & 0.19 & 3.98 & 2529 & 179 & 2718 & 66 & 2635 & 89 \\
\hline $06 \mathrm{Z4}$ & 0.31 & 0.54 & 8.48 & 13.83 & 9.49 & 0.19 & 4.25 & 2771 & 191 & 2713 & 70 & 2738 & 90 \\
\hline $09 \mathrm{Z5}$ & 0.28 & 0.49 & 10.29 & 12.59 & 11.37 & 0.19 & 4.83 & 2584 & 219 & 2700 & 80 & 2650 & 107 \\
\hline $10 \mathrm{Z6}$ & 0.16 & 0.50 & 9.57 & 12.69 & 10.66 & 0.19 & 4.69 & 2593 & 204 & 2706 & 77 & 2657 & 100 \\
\hline $11 \mathrm{Z7}$ & 0.26 & 0.50 & 9.79 & 12.88 & 10.76 & 0.19 & 4.48 & 2631 & 211 & 2700 & 74 & 2671 & 101 \\
\hline $12 \mathrm{Z} 8$ & 0.55 & 0.42 & 10.46 & 11.25 & 11.31 & 0.19 & 4.30 & 2263 & 200 & 2775 & 71 & 2544 & 105 \\
\hline $16 \mathrm{Z9}$ & 0.33 & 0.52 & 7.47 & 13.30 & 8.52 & 0.19 & 4.11 & 2696 & 164 & 2705 & 68 & 2701 & 80 \\
\hline $17 \mathrm{Z} 10$ & 0.28 & 0.51 & 7.58 & 13.20 & 8.59 & 0.19 & 4.05 & 2646 & 164 & 2730 & 67 & 2694 & 81 \\
\hline $18 \mathrm{Z} 11$ & 0.40 & 0.53 & 9.34 & 13.81 & 10.16 & 0.19 & 3.99 & 2760 & 210 & 2720 & 66 & 2737 & 96 \\
\hline $21 \mathrm{Z12}$ & 0.23 & 0.52 & 11.28 & 13.28 & 12.27 & 0.18 & 4.83 & 2703 & 249 & 2697 & 80 & 2699 & 116 \\
\hline $22 \mathrm{Z} 13$ & 0.12 & 0.46 & 7.93 & 10.82 & 9.01 & 0.17 & 4.27 & 2434 & 161 & 2568 & 71 & 2508 & 84 \\
\hline $23 \mathrm{Z14}$ & 0.27 & 0.52 & 8.90 & 13.38 & 9.83 & 0.19 & 4.17 & 2710 & 197 & 2704 & 69 & 2707 & 93 \\
\hline $24 \mathrm{Z15}$ & 0.29 & 0.50 & 9.72 & 12.61 & 10.56 & 0.18 & 4.14 & 2610 & 209 & 2682 & 68 & 2651 & 99 \\
\hline $27 \mathrm{Z16}$ & 0.33 & 0.52 & 7.24 & 13.18 & 8.39 & 0.19 & 4.23 & 2681 & 159 & 2701 & 70 & 2692 & 79 \\
\hline $28 \mathrm{Z19}$ & 0.28 & 0.50 & 7.98 & 12.55 & 9.00 & 0.18 & 4.15 & 2592 & 170 & 2688 & 69 & 2647 & 85 \\
\hline $29 \mathrm{Z} 18$ & 0.23 & 0.54 & 8.43 & 13.72 & 9.31 & 0.19 & 3.97 & 2771 & 190 & 2701 & 66 & 2731 & 88 \\
\hline $30 \mathrm{Z19}$ & 0.42 & 0.54 & 9.67 & 13.73 & 10.45 & 0.19 & 3.95 & 2768 & 218 & 2704 & 65 & 2731 & 99 \\
\hline $33 \mathrm{Z} 20$ & 0.15 & 0.42 & 6.80 & 8.69 & 8.01 & 0.15 & 4.23 & 2248 & 129 & 2358 & 72 & 2306 & 73 \\
\hline $15 \mathrm{UQZ}$ & 0.08 & 0.19 & 7.69 & 2.03 & 9.43 & 0.08 & 5.47 & 1132 & 80 & 1110 & 109 & 1124 & 64 \\
\hline $34 \mathrm{UQZ}$ & 0.09 & 0.20 & 7.96 & 2.11 & 9.39 & 0.08 & 4.99 & 1165 & 85 & 1127 & 99 & 1152 & 65 \\
\hline
\end{tabular}

Suíte Jussiape

\begin{tabular}{c|c|c|c|c|c|c|c|c|c|c|c|c|c}
\hline \multicolumn{10}{c}{ Amostra SCP-SJ-01 } \\
\hline 03 Z1 & 0.32 & 0.43 & 4.30 & 10.40 & 6.10 & 0.17 & 13.00 & 2322 & 166 & 2595 & 202 & 2471 & 87 \\
\hline 04 Z2 & 0.08 & 0.31 & 3.80 & 5.09 & 5.46 & 0.12 & 11.84 & 1730 & 115 & 1955 & 198 & 1835 & 74 \\
\hline 05 Z3 & 0.27 & 0.40 & 5.59 & 8.70 & 7.92 & 0.16 & 16.88 & 2150 & 201 & 2449 & 261 & 2307 & 109 \\
\hline 06 Z4 & 0.20 & 0.48 & 3.70 & 12.16 & 5.22 & 0.18 & 11.12 & 2515 & 152 & 2696 & 173 & 2617 & 75 \\
\hline 09 Z5 & 0.13 & 0.18 & 6.29 & 2.96 & 8.88 & 0.09 & 20.18 & 1080 & 124 & 1484 & 341 & 1396 & 103 \\
\hline 10 Z6 & 0.60 & 0.48 & 4.88 & 12.37 & 6.91 & 0.19 & 14.41 & 2548 & 202 & 2699 & 220 & 2633 & 95 \\
\hline 11 Z7 & 0.07 & 0.38 & 5.87 & 6.99 & 8.38 & 0.13 & 17.20 & 2080 & 206 & 2139 & 273 & 2110 & 106 \\
\hline 12 Z8 & 0.09 & 0.23 & 8.11 & 3.71 & 12.33 & 0.12 & 24.00 & 1313 & 194 & 1941 & 377 & 1573 & 130 \\
\hline 15 Z9 & 0.02 & 0.29 & 4.36 & 4.93 & 6.28 & 0.12 & 13.47 & 1661 & 127 & 1980 & 222 & 1807 & 83 \\
\hline 16 Z10 & 0.00 & 0.26 & 5.73 & 4.34 & 8.15 & 0.12 & 16.89 & 1469 & 149 & 1999 & 273 & 1700 & 97 \\
\hline 17 Z11 & 0.18 & 0.38 & 4.35 & 7.26 & 6.23 & 0.12 & 14.87 & 2114 & 156 & 1903 & 246 & 2144 & 99 \\
\hline 18 Z12 & 0.03 & 0.37 & 5.92 & 6.43 & 8.44 & 0.13 & 17.41 & 2010 & 201 & 2063 & 279 & 2036 & 106 \\
\hline 21 Z13 & 0.92 & 0.23 & 4.74 & 4.23 & 7.37 & 0.13 & 15.03 & 1331 & 116 & 2146 & 241 & 1679 & 90 \\
\hline 22 Z14 & 0.07 & 0.61 & 4.15 & 20.28 & 6.01 & 0.24 & 12.72 & 3084 & 200 & 3118 & 189 & 3105 & 89 \\
\hline 23 Z15 & 0.02 & 0.50 & 4.12 & 8.96 & 5.95 & 0.13 & 12.68 & 2599 & 174 & 2100 & 207 & 2334 & 84 \\
\hline 24 UQZ & 0.14 & 0.19 & 6.14 & 2.12 & 8.74 & 0.07 & 18.64 & 1152 & 128 & 1068 & 335 & 1154 & 91 \\
\hline 27 Z16 & 0.04 & 0.42 & 4.54 & 7.42 & 6.56 & 0.13 & 13.87 & 2272 & 172 & 2060 & 226 & 2163 & 90 \\
\hline 28 Z17 & 0.31 & 0.33 & 4.05 & 5.82 & 5.96 & 0.13 & 12.65 & 1850 & 130 & 2055 & 208 & 1949 & 81 \\
\hline 29 Z18 & 0.04 & 0.52 & 3.64 & 13.16 & 5.29 & 0.18 & 11.30 & 2701 & 159 & 2680 & 176 & 2691 & 78 \\
\hline 30 Z19 & 0.02 & 0.38 & 5.76 & 6.43 & 8.20 & 0.12 & 17.03 & 2066 & 201 & 2000 & 275 & 2036 & 104 \\
\hline 33 Z20 & 0.01 & 0.38 & 3.71 & 6.51 & 5.40 & 0.13 & 11.50 & 2058 & 130 & 2034 & 190 & 2047 & 74 \\
\hline 34 UQZ & 0.10 & 0.20 & 5.03 & 2.12 & 7.22 & 0.07 & 15.85 & 1176 & 107 & 1063 & 290 & 1157 & 80 \\
\hline & & & & & & & & & & & & \\
\hline
\end{tabular}




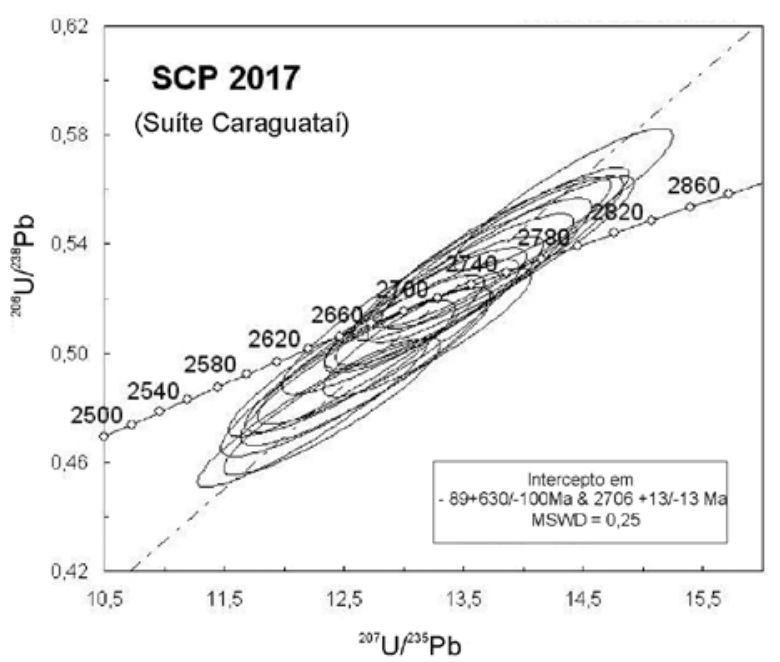

Figura 6 - Diagrama Concórdia U-Pb para o Augen mesomilonito do Suite Caraguataí.

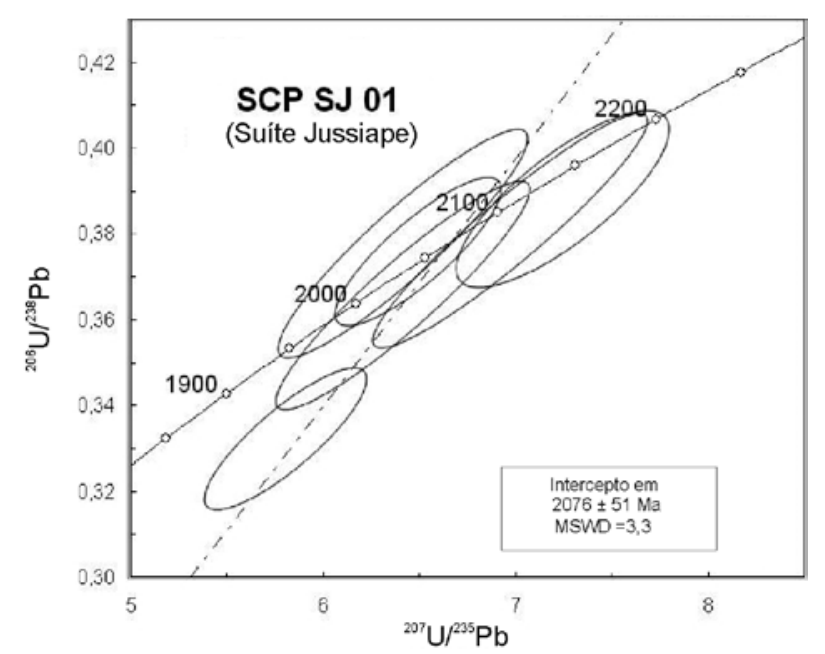

Figura 7 - Diagrama U/Pb para a amostra do Granito Jussiape.

pesados usando técnicas gravimétricas e magnéticas convecionais. Em seguida, foram encaminhadas ao Laboratório de Geocronologia da Universidade de Brasília para serem submetidas à análise isotópica utilizando os equipamentos LA-ICPMS (U-Pb) de acordo com as metodologias descritas em Buhn et al. (2009) e Gioia \& Pimentel (2000), respectivamente.

Resultados U-Pb (LA-ICPMS) da Suíte Caraguataí Uma amostra de quartzo-sienito milonítico da Suíte Caraguataí foi analisada isotopicamente pelo método U-Pb (LA-ICPMS). De acordo com os resultados isotópicos apresentados na tabela 2 e na figura 6, a idade U-Pb encontrada para essas rochas foi $2711 \pm 13$ e pode ser interpretada como a época de cristalização do protólito. Essa idade $\mathrm{U}-\mathrm{Pb}$ reflete, possivelmente, uma granitogênese anorogênica associada com rifteamentos de uma crosta arqueana que, posteriormente, foi reciclada nos eventos tectônicos do Paleoproterozóico. Regionalmente, conforme observado na tabela 1 , idades U-Pb similares foram encontradas para os granitos alcalinos da serra do Eixo (Pinto et al. 1998) e Guajeru (Lopes 2002). Da mesma forma, datações radiométricas obtidas por outras metodologias geocronológicas (vide tabela 1) situam-se nessa mesma época. Esse é o caso das idades $\mathrm{Rb}-\mathrm{Sr}$ do Gnaisse Piripá e do Tonalito Bernada e das idades modelos Sm-Nd $\left(\mathrm{T}_{\mathrm{DM}}\right)$ dos granitos Lagoa Grande/Lagoinha (Marinho 1991, Nutman et al. 1993); Rio do Paulo e Caculé (Leal et al. 2000); Gameleira (Sabaté et al. 1990, Marinho 1991) e do Complexo Lagoa Real (Cordani et al. 1992). Esses dados, em conjunto, sugerem que a fusão de fontes paleoarqueanas geradoras do magmatismo neoarqueano são evidentes e caracterizam este como um importante evento de formação de crosta continental da região (Cordani et al. 1992, Marinho 1991, Leal 1998).

Resultados U-Pb (LA-ICPMS) da Suíte Jussiape Para a amostra do Granito Jussiape foi encontrada uma idade U-Pb de $2076 \pm 51 \mathrm{Ma}$ (Tab. 2, Fig. 7). De fato, as relações de campo sugerem que as rochas da Suíte Jussiape são intrusivas nas unidades da Suíte Caraguataí. Por outro lado, essa idade é próxima àquelas idades $\mathrm{U}-\mathrm{Pb}$ dos granitoides Veredinha $(2103$ $\pm 11 \mathrm{Ma}$; Arcanjo et al. 2000) e Ibitiara (2091 $\pm 6 \mathrm{Ma}$; Guimarães et al. 2005) e de outra amostra do Granito Jussiape (2122 2 Ma; Guimarães et al. 2005). Além disso, Leal et al. (2000) também obteve um conjunto de idades $\mathrm{Pb}-\mathrm{Pb}$ para os granitóides do Bloco Gavião. Esse é o caso, por exemplo, dos granitos Caculé $(2019 \pm 32$ $\mathrm{Ma})$ e Espírito Santo (2012 $\pm 25 \mathrm{Ma})$. Essas idades Pb$\mathrm{Pb}$ são muito próximas àquelas idades $\mathrm{Rb}-\mathrm{Sr}$ obtidas no Granito de Iguatemi pelos mesmos autores (Tab. 1).

CONCLUSÕES A Suíte Caraguataí compreende álcali-feldspato granitos, sienitos/quartzo-sienitos, leuco a mesocráticos, de coloração cinza, que foram submetidos a variados estágios de deformação em zonas de cisalhamento, gerando protomilonitos, augen-mesomilonitos e ultramilonitos. A idade U-Pb (LA-ICPMS) da Suíte Caraguataí para a amostra analisada sugere cristalização do protólito em $2711 \pm 13$. Esse dados sugere a existência de um magmatismo neoarqueano nesse setor do Bloco Gavião e demonstram a importância de um evento magmático dessa idade. O granito Jussiape, por sua vez, intrusiva na primeira, compreende monzogranitos a sienogranitos, milonitizados ou não, hololeucocráticos a leucocráticos, de coloração rósea. A única amostra analisada dessa suíte revelou idade de cristalização em torno de $2076 \pm$ $51 \mathrm{Ma}$, associada com um magmatismo riaciano.

Agradecimentos Os autores querem expressar seus agradecimentos ao $\mathrm{CNPq}$ pelas bolsas de PósDoutorado Júnior de Simone C. P. Cruz (Processo 150127/2005-7) e de pesquisa de Mauricio Antônio 
Carneiro, assim como pelos recursos disponibilizados através do Edital Universal (Processo 475092/20040 ). Além disso, agradecem à Companhia Baiana de
Pesquisa Mineral (CBPM) pelo apoio nas campanhas de campo e a FAPEMIG pelos projetos CRA 00281-09; 5118-5.0207; 2032-05.

\section{Referências}

Arcanjo J.B., Marques-Martins A.A., Loureiro H.S.C., Varela P.H.L. 2000. Projeto vale do Paramirim, escala 1:100.000. Programa de Levantamentos Geológicos Básicos do Brasil, Cd-Room.

Babinski M., Pedreira A., Brito-Neves B.B., Van-Schmus W.R. 1999. Contribuição à geocronologia da Chapada Diamantina. In: SBG, Simpósio Nacional de Estudos Tectônicos, 7, Anais, p. 118-121.

Barbosa J.S.F \& Dominguez J.M.L (eds.) 1996. Mapa Geológico do Estado da Bahia. Escala: 1.000.000. Texto explicativo, Salvador, 382 p.

Barbosa J.S.F. \& Sabaté P. 2002. Geological feature and the paleoproterozoic od four archean crustal segments of the São Francisco Craton, Bahia, Brazil. A syntesis. Anais da Academia Brasileira de Ciências, 2:343-359.

Buhn B., Pimentel M.M., Matteini M., Dantas E.L. 2009. High spatial resolution analysis of $\mathrm{Pb}$ and $\mathrm{U}$ isotopes for geochronology by laser ablation multi-collector inductively coupled plasma mass spectrometry (LAMC-ICP-MS). Anais da Acad. Bras. de Ciências, 1:1-16.

Cordani U.G. \& Iyer S.S. 1979 Geocronological investigation on the Precambrian Granulitic terrain of Bahia, Brazil. Precambrian Research, 9:255-257.

Cordani U.G., Sato K., Marinho M.M. 1985. The geologic evolution of the ancient granite-greenstone terrane of central-southern Bahia, Brazil. Precambrian Research, 27: 187-213.

Cordani U.G., Iyer S.S., Taylor P.N., Kawashita K., Sato K., Mcreath I. 1992. Pb-Pb, Rb-Sr, and K-Ar sistematic of the Lagoa Real uranium province (south-central Bahia, Brazil) and the Espinhaço Cycle (ca. 1.5-1.0 Ga). Journal of South American Earth Science, 1:33-46.

Cordani U.G., Sato K., Coutinho J.M., Nutman A. 1997. Geocronological Interpretation in areas with complex evolution: The case of Piripá, central-southern Bahia, Brazil. In: South American Symposium on Isotope Geology, Extended Abstracts, p.85-87.

Cruz S.C.P. 2004. A Interação Tectônica entre o Aulacógeno do Paramirim e o Orógeno Araçuaí - Oeste Congo. Tese Doutoramento, Univ. Federal de Ouro Preto, 505 p.

Cruz S.C.P., Barbosa J.S.F., Alves E.S., Damasceno G.C., Machado G.S., Borges J.O., Gomes A.M., Mesquita L., Pimentel I., Leal A.B.M., Palmeira D.S. 2009. Mapeamento geológico e levantamentos de recursos minerais da olha Caetité (escala 1:100.000). Programa de Levantamentos Geológicos Básicos, Convênio UFBA-CPRM-FAPEX, Salvador, 175 p.

Cunha J.C. \& Fróes R.J.B. 1994. Komatítos com textura spinifex do Greenstone Belt de Umburanas, Bahia. Salvador, CBPM, 29p.

Cunha J.C., Leal L.R., Fróes R.J.B., Teixeira W., Macambira M.J.B. 1996. Idade dos greenstone belts e dos terrenos TTG's associados da região de Brumado, centro oeste do Cráton do São Francisco (Bahia-Brasil). In: SBG, Congresso Brasileiro de Geologia, 39, Anais, p. 67-70.

Danderfer A., De Waele B., Pedreira A., Nalini H.A. 2009. New geochronological constraints on the geological evolution of Espinhaço basin within the São Francisco Craton-Brazil. Precambrian Research, 170:116-128.

Gioia S.M. \& Pimentel M.M. 2000. The Sm-Nd isotopic method in the geochronology laboratory of the University of Brasília. Anais da Acad. Bras. de Ciências, 2:219-245.

Guimarães J.T. 1996. A Formação Bebedouro no Estado da Bahia: Faciologia, Estratigrafia e Ambiente de Sedimentação. Dissertação de Mestrado, Instituto de Geociências, Universidade Federal da Bahia, 155 p.

Guimarães J.T., Teixeira L.R., Silva M.G. Martins A.A.M., Filho E.L.A., Loureiro H.S.C., Arcanjo J.B., Dalton de Souza J., Neves J.P., Mascarenhas J.F., Melo R.C., Bento R.V. 2005. Datações U-Pb em rochas magmáticas intrusivas no Complexo Paramirim e no Rifte Espinhaço: Uma contribuição ao estudo da Evolução Geocronológica da Chapada Diamantina. In: SBG/BA-SE, Simpósio do Cráton do São Francisco, Salvador, Anais de Resumos Expandidos, p. 159-161.

Leal L.R.B., Teixeira W., Cunha J.C., Macambira M.J.B. 1997. Crustal evolution of Gavião block of the São Francisco Craton: A geochronological study with, $\mathrm{Pb}-\mathrm{Pb}, \mathrm{Sm}-\mathrm{Nd}, \mathrm{Rb}-\mathrm{Sr}$ and K-Ar. In: South American Symposium on Isotope Geology, 2, Extended Abstract, p. 161-162.

Leal L.R.B. 1998. Geocronologia U-Pb (SHRIMP), ${ }^{207} \mathrm{~Pb} /{ }^{206} \mathrm{~Pb}, \mathrm{Rb}-\mathrm{Sr}$, Sm-Nd e K-Ar dos Terrenos GranitoGreenstone do Bloco do Gavião: Implicações para Evolução arqueana e proterozóica do Cráton do São Francisco, Brasil. Tese de Doutoramento, Instituto de Geociências, Universidade Estado de São Paulo, 178 p.

Leal L.R.B, Teixeira W., Cunha J.C., Macambira M.J.B. 1998. Archean tonalitic-trondhjemitic and granitic plutonism in the Gavião Block, São Francisco Craton, Bahia, Brazil: Geochemical and geochronology characteristics. Revista Brasileira de Geociências, 2:209-220.

Leal L.R.B., Teixeira W., Cunha J.C., Leal A.B.M., Macambira M.J.B., Rosa M.L.S. 2000. Isotopic signatures of paleoproterozoic granitoids of the Gavião block and implications for the evolution of the São Francisco craton, Bahia, Brazil. Revista Brasileira de Geociências, 30:066-069.

Leal A.B.M., Leal L.R.B., Cunha J.C., Leal L.R.B., Cunha J.C., Cordani U.G., Teixeira W., Nutman A.P., Leal A.B.M., Macambira M.J.B. 2003. Shrimp U-Pb, $207 \mathrm{~Pb} / 206 \mathrm{~Pb}$ zircon dating, and $\mathrm{Nd}$ isotopic signature of the Umburanas Greenstone Belt, northern São Francisco Craton, Brazil. Journal of South American Earth Sciences, 15:775-785.

Ledru P., Cocherie A., Barbosa J.S.F., Johan V., Onstott T. 
1993. Âge du Métamorphisme granulitique dans Le Craton Du São Francisco (Brésil): Inplications Sur la nature de lórogene transmazoneen. C. R. Acad. Sci. Paris, 211:120-125.

Lopes G.A.C. 1991. Investigação da metalogênese de granitóides da região de Vitória da Conquista. SME/ CBPM, volume 1, textos e mapas, $70 \mathrm{p}$.

Lopes G.A.C. 2002. Projeto Guajeru. Salvador, CBPM, v.1, $408 \mathrm{p}$.

Loureiro H.S.C., Lima E.S., Macedo E.P., Silveira F.V., Bahiense I.C., Arcanjo J.B.A., Moraes-Filho J.C., Neves J.P., Guimaraes J.T., Rodrigues L.T., Abram M.B., Santos R. A., Melo R.C. 2010. Geologia e Recursos Mineris da Parte norte do Corredor de Deformação do Paramirim: Projeto Barra-Oliveira dos Brejinhos. Salvador: CBPM, 118 p. (il. Série Arquivos Abertos, 33).

Marinho M.M., Costa P.H., Silva E.FA., Torquato J.R. 1979. Evolução Geotectônica do Pré-cambriano no Estado da Bahia. In: Inda H.A.V. (eds.) Geologia e recursos minerais do Estado da Bahia; textos básicos. Salvador, SME/CPM, p. 57-157.

Marinho M.M. 1991. Lê sequence Vulcano-Sedimentaire de Contendas Mirante et la Bordure Ocidentale du Bloc de Jequié (Craton du São Francisco, Brésil): um example de transition Archeen-Proterozoic. Doctor of Philosophy Thesis, Universidade de Clemont-Ferrand, $257 \mathrm{p}$.

Marinho M.M., Rios D.C., Conceição H., Rosa M.L.S. 2008. Magmatismo alcalino neoarqueano no Cráton do São Francisco, Bahia: pluton Pé de Serra. In: SBG, Congresso Brasileiro de Geologia, 44, Anais, p. 57.

Martin H., Peucat J.J., Sabaté P., Cunha J.C. 1991. Um segment de croûte continentale d'Age archéean ancien (3.5 millards d'années): lê massif de Sete Voltas (Bahia, Brésil). C.R. Acad. Sci. Phis., 313:531-538.

Martin H., Peucat J.J., Sabaté P., Cunha J.C. 1997. Crustal evolution in early archean of south América: Exemple of Sete Voltas Massif, Bahia State, Brazil. Precambrian Research, 82:35-62.

Mascarenhas J.F. \& Garcia T.M. 1989. Mapa geocronológico do estado da Bahia. Secretaria de Minas e Energia, Texto Explicativo, Salvador, Bahia, 130 p.

Nutman A.P. \& Cordani U.G. 1993. Shrimp U-Pb zircon geochronology of Archean granitoids from the Contendas Mirante area of the São Francisco Craton, Bahia, Brazil. Journal of South American Earth Science, 7:107-114.

Nutman A.P., Cordani U.G., Sabaté P. 1993. Shrimp $\mathrm{U}-\mathrm{Pb}$ ages of detritical zircons from early Proterozoic Contendas Mirantes area of the São Francisco Craton, Bahia, Brazil. Precambrian Research 63:179-188.

Peucat J.J., Mascarenhas J.F., Barbosa J.S.F., de Souza S.L., Marinho M.M., Fanning C.M., Leite C.M.M. 2002. 3.3 $\mathrm{Ga}$ SHRIMP U-Pb zircon age of a felsic metavolcanic rock from the Mundo Novo Greenstone belt in the São Francisco Craton, Bahia (NE Brazil). South American Journal of Earth Sciences, 15:363-373.

Pimentel M.M., Machado N., Lobato L.M. 1994. Geocronologia $\mathrm{U}-\mathrm{Pb}$ de rochas graníticas e gnáissicas da região de Lagoa Real, Bahia, e implicações para a idade da mineralização de urânio. In: SBG, Congresso
Brasileiro de Geologia, 38, Boletin de Resumos Expandidos, p. 389-390.

Pinto M.A. 1996. Le recyclage de la croúte continentale archéenne: Exemple du bloc du Gavião-Bahia, Bresil. Doctor of Philosophy Thesis, Geociences Rennes, 193 p.

Pinto M.A.S., Peucat J.J., Martin H., Sabaté P. 1998. Recycling of the Archaean continental crust: the case study of the Gavião Block, Bahia, Brazil. Journal of South American Earth Science, 5:487-498.

Rosa M.L.S., Conceição H., Paim M.M., Santos E.B., Alves F.C. Leahy G.S., Leal L.R. 1996. Magmatismo potássico/ultrapotássico Pós a tardi orogênico associado à subducção no oeste da Bahia: Batólito Monso-sienitico de Guanambi-Urandi e os sienitos de Correntina. Geochimica Brasiliensis, 1:027-042.

Sabaté P., Marinho M.M., Vidal P., Vauchette M. 1990. The 2-Ga peraluminous magmatism of the JacobinaContendas Mirantes Belts (Bahia, Brazil): Geologic and isotopic constraints on the sources. Chemical Geology, 83:325-338.

Sato K. 1998. Evolução crustal da plataforma sul americana, com base na geoquímica Isotópica SM-Nd. Tese de Doutoramento, Instituto de Geociências, Universidade de São Paulo, 297 p.

Silva M.G. \& Cunha J.C. 1999. Greenstone Belts and equivalent volcano-sedimentary sequences of the São Francisco Craton, Bahia, Brazil, Brazil - Geology and Mineral Potential. In: Silva M.G. \& Misi A. Base Metal deposits of Brazil. MME/CPRM/DNPM, Belo Horizonte, p. 92-99.

Schobbenhaus C., Hoppe A., Baumann A., Lork A. 1994. Idade $\mathrm{U} / \mathrm{Pb}$ do vulcanismo Rio dos Remédios, Chapada Diamantina, Bahia. In: SBG, Congresso Brasileiro de Geologia, 38, Anais, 2, p. 397-399.

Sabaté P., Marinho M.M., Vidal P., Vauchette M. 1990. The 2-Ga peraluminous magmatism of the JacobinaContendas Mirantes Belts (Bahia, Brazil): Geologic and isotopic constraints on the sources. Chemical Geology, 83:325-338.

Teixeira L. 2005. Relatório Temático de Litogeoquímica: Projeto Ibitiara-Rio de Contas. Salvador, CPRM/CBPM, $51 \mathrm{p}$.

Turpin L., Maruèjol P., Cuney M. 1988. U-Pb, Rb-Sr and Sm-Nd chronology of granitic basement, hydrotermal albitites and uranium mineralization, Lagoa Real, South Bahia, Brazil. Contrib. Mineral. Petrol., 98:139-147.

Teixeira W. 2005. Características geoquímicas dos granitóides transamazônicos no Bloco Gavião, Craton São Francisco, Bahia, Brasil. Geochimica Brasiliensis, 19:8-21.

Wilson N., Moorbath S., Taylor P.N., Barbosa J. 1988. Archean and early Proterozoic crustal evolution in the São Francisco Craton, Bahia, Brazil. Chemical Geology, 70:147.

Manuscrito ID 15165 Submetido em 24 de agosto de 2009 Aceito em 26 de maio de 2011 